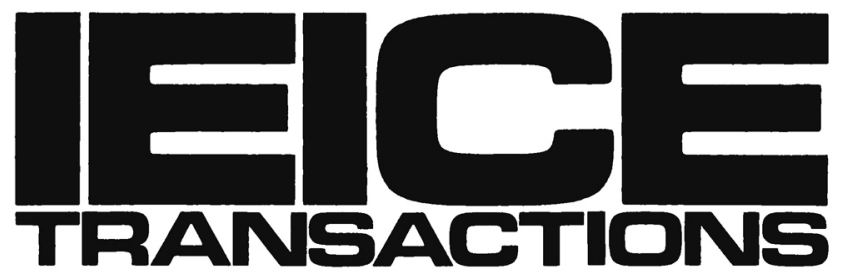

\title{
on Communications
}

VOL. E100-B NO. 6

JUNE 2017

The usage of this PDF file must comply with the IEICE Provisions on Copyright.

The author(s) can distribute this PDF file for research and educational (nonprofit) purposes only.

Distribution by anyone other than the author(s) is prohibited. 


\title{
PAPER \\ Precoding Design for Han-Kobayashi's Signal Splitting in MIMO Interference Networks
}

\author{
Ho Huu Minh TAM ${ }^{\dagger a)}$, Hoang Duong TUAN ${ }^{\dagger b)}$, Duy Trong NGO ${ }^{\dagger \dagger c)}$, Nonmembers, \\ and Ha Hoang NGUYEN ${ }^{\dagger \dagger \dagger \mathrm{d})}$, Member
}

\begin{abstract}
SUMMARY For a multiuser multi-input multi-output (MU-MIMO) multicell network, the Han-Kobayashi strategy aims to improve the achievable rate region by splitting the data information intended to a serviced user (UE) into a common message and a private message. The common message is decodable by this UE and another UE from an adjacent cell so that the corresponding intercell interference is cancelled off. This work aims to design optimal precoders for both common and private messages to maximize the network sum-rate, which is a highly nonlinear and nonsmooth function in the precoder matrix variables. Existing approaches are unable to address this difficult problem. In this paper, we develop a successive convex quadratic programming algorithm that generates a sequence of improved points. We prove that the proposed algorithm converges to at least a local optimum of the considered problem. Numerical results confirm the advantages of our proposed algorithm over conventional coordinated precoding approaches where the intercell interference is treated as noise.

key words: interference mitigation, interference networks, nonconvex optimization, precoding design, successive convex quadratic programming
\end{abstract}

\section{Introduction}

There is limited understanding of the capacity of interference networks (INs). By treating residual interference as noise, the network capacity is achieved only at low interference regime (see [1] and references therein) for a general multi-user IN or at certain sufficient conditions in terms of matrix equations for two-user INs [2]. For a two-user twocell IN (i.e., one user per cell), the Han-Kobayashi (H-K) strategy [3] is known to give the best achievable rate region [4], [5]. With the H-K strategy, the transmitted data information of both users is split into two parts: a private message to be decoded at the intended receiver and a common message that can be decoded at both receivers. A part of the interference is thus cancelled off by decoding the common message, while the remaining private message from the other user is

Manuscript received May 19, 2016.

Manuscript revised October 11, 2016.

Manuscript publicized December 14, 2016.

The authors are with the Faculty of Engineering and Information Technology, University of Technology, Sydney, NSW 2007, Australia.

${ }^{\dagger}$ The author is with School of Electrical Engineering and Computer Science, The University of Newcastle, Callaghan, NSW 2308, Australia.

${ }^{+1 \dagger}$ The author is with Department of Electrical and Computer Engineering, University of Saskatchewan, Saskatoon, SK S7N 5A9, Canada.

a) E-mail: huuminhtam.ho@student.uts.edu.au

b) E-mail: tuan.hoang@uts.edu.au

c) E-mail: duy.ngo@newcastle.edu.au

d)E-mail: ha.nguyen@usask.ca

DOI: 10.1587/transcom.2016EBP3209 treated as noise. Accordingly, it is challenging to perform constructive optimization over such an achievable rate region to realize the potential of H-K strategy [4], [6].

Jointly beamforming common and private messages to maximize the achievable rate across multiuser multi-input single-output (MU-MISO) INs is first considered in [7]. At discrete points of the joint space of common and private rates, an ad-hoc intensive search is carried by rank-one constrained semi-definite programming (SDP) for the beamformer vectors. Still, the optimal rate is not achieved. Furthermore, the search proposed by [7] is not suitable for the problem of sum-rate maximization, which is a more popular metric for INs. Inspired by [7], the works of [8], [9] design covariance matrices of the common and private messages in MU-MIMO multicell INs and beamformers for such messages in MU-MISO multicell INs to maximize either the sum-rate or the achievable rate across the networks.

Our present work aims to find optimal precoder matrices for the common and private messages of independent data streams. The objective is to maximize the sum-rate of an MU-MIMO multicell network. The available solution approaches are not applicable, e.g., [10] for coordinated precoding private messages only and [8], [9] for covariance design. We propose a successive optimization algorithm in which each iteration only solves a simple convex quadratic program of low computational complexity. Once initialized from a feasible point, our algorithm generates a sequence of monotonically improved points, which eventually converge to at least a local maximum of the formulated nonconvex and nonsmooth problem.

The rest of this paper is organized as follows: Section 2 presents the system model and formulates the precoder design problem. Section 3 proposes the successive quadratic programming algorithm for solution. Section 4 verifies the advantages of our devised solution by numerical examples.

Notations. $\mathbf{I}_{n}$ is the identity matrix of size $n \times n$. The notation $(\cdot)^{H}$ stands for the Hermitian transpose. The inner product $\langle\mathbf{X}, \mathbf{Y}\rangle$ is defined as $\operatorname{trace}\left(\mathbf{X}^{H} \mathbf{Y}\right)$. $\langle\mathbf{A}\rangle$ denotes the trace of a matrix $\mathbf{A}$, and $|\mathbf{A}|$ denotes the determinant of a square matrix A. For Hermitian symmetric matrices $\mathbf{A}$ and $\mathbf{B}$, the notation $\mathbf{A} \geq \mathbf{B}$ ( $\mathbf{A}>\mathbf{B}$, respectively) means that $\mathbf{A}-$ $\mathbf{B}$ is a positive semidefinite (positive definite, respectively) matrix. $\mathbb{E}\{$.$\} denotes the expectation operator, \mathbb{C}$ is the set of all complex numbers, and $\emptyset$ is an empty set. $\mathfrak{R}\{x\}$ denotes the real part of a complex number $x$. 


\section{System Model and Problem Formulation}

Consider the downlink transmissions in a network consisting of $N$ cells, where the base station (BS) of each cell is equipped with $N_{t}$ antennas and it serves $K$ UEs within its cell. Each UE is equipped with $N_{r}$ antennas. Upon denoting $\mathcal{I} \triangleq\{1,2, \ldots, N\}$ and $\mathcal{J} \triangleq\{1,2, \ldots, K\}$, the $j$-th UE in the $i$-th cell is referred as $\operatorname{UE}(i, j) \in \mathcal{S} \triangleq \mathcal{I} \times \mathcal{J}$. Signals are precoded at the BSs prior to transmitting to the UEs. To implement the H-K strategy, where each user decodes the common message of at most one other user, we follow [7], [8] and introduce the pairing operator $a(i, j)$ to specify which other UE, beside UE $(i, j)$ itself, decodes the common message of UE $(i, j)$. When $\mathrm{UE}(i, j)$ has no common message, we let $a(i, j)$ be an empty set. Formally, it is a mapping $a: \mathcal{I} \times \mathcal{J} \rightarrow(\mathcal{I} \times \mathcal{J}) \cup\{\emptyset\}$ with the restriction that $a(i, j)=(\tilde{i}, \tilde{j})$ always has $\tilde{i} \neq i$ and $a^{-1}(\tilde{i}, \tilde{j})$ has cardinality of no more than one. With $\emptyset \neq a(i, j)=(\tilde{i}, \tilde{j}), \tilde{i} \neq i$, UE $(i, j)$ may split its $L\left(\leq N_{t}\right)$ data streams into two parts: the private message $\mathbf{s}_{i, j}^{p} \in \mathbb{C}^{L}$ with $\mathbb{E}\left\{\mathbf{s}_{i, j}^{p}\left(\mathbf{s}_{i, j}^{p}\right)^{H}\right\}=\mathbf{I}_{L}$, and the common message $\mathbf{s}_{i, j}^{c} \in \mathbb{C}^{L}$ with $\mathbb{E}\left\{\mathbf{s}_{i, j}^{c}\left(\mathbf{s}_{i, j}^{c}\right)^{H}\right\}=\mathbf{I}_{L}$. The private and common messages are precoded by matrices $\mathbf{V}_{i, j}^{p} \in \mathbb{C}^{N_{t} \times L}$ and $\mathbf{V}_{i, j}^{c} \in \mathbb{C}^{N_{t} \times L}$, respectively. The common message $\mathbf{s}_{i, j}^{c}$ of $\mathrm{UE}(i, j)$ is to be decoded by UE $(i, j)$ 's receiver and also by UE $(\tilde{i}, \tilde{j})$ 's receiver in a different cell $\tilde{i}$. On the other hand, if $(i, j)=a(\hat{i}, \hat{j})$ for some $\hat{i} \neq i$, the receiver of $\operatorname{UE}(i, j)$ also decodes the common message $\mathbf{s}_{\hat{i}, \hat{j}}^{c}$ from UE $(\hat{i}, \hat{j})$ in a different cell $\hat{i} \neq i$.

As in [7], [8], each UE $(i, j)$ successively decodes the following messages (in the following strict order): $(a)$ its common message $\mathbf{s}_{i, j}^{c}$ from its own transmitter; $(b)$ the common message $\mathbf{s}_{\hat{i}, \hat{j}}^{c}$ from UE $(\hat{i}, \hat{j})$ 's transmitter in the different cell $\hat{i} \neq i$ for which $a(\hat{i}, \hat{j})=(i, j) ;(c)$ the private message $\mathbf{s}_{i, j}^{p}$ from its own transmitter. Note that the decoded messages are also successively subtracted from the received signal for interference mitigation. Intuitively, one's own common message is decoded first to help the decoding of the common information from the other transmitter, while its own private message is decoded last to take advantage of the reduced interference due to common message decoding.

For notational convenience, let us define $\mathbf{V}_{i, j} \triangleq$ $\left[\begin{array}{ll}\mathbf{V}_{i, j}^{p} & \mathbf{V}_{i, j}^{c}\end{array}\right], \mathbf{V} \triangleq\left[\mathbf{V}_{i, j}\right]_{(i, j) \in \mathcal{S}}, \mathbf{s}_{i, j} \triangleq\left[\begin{array}{c}\mathbf{s}_{i, j}^{p} \\ \mathbf{s}_{i, j}^{c}\end{array}\right]$. The received signal at $\mathrm{UE}(i, j) \in \mathcal{S}$ is expressed as:

$$
\mathbf{y}_{i, j}=\sum_{(s, l) \in \mathcal{S}} \mathbf{H}_{s, i, j} \mathbf{V}_{s, l} \mathbf{s}_{s, l}+\mathbf{n}_{i, j},
$$

where $\mathbf{H}_{s, i, j} \in \mathbb{C}^{N_{r} \times N_{t}}$ is the matrix of channel coefficients from BS $s \in \mathcal{I}$ to UE $(i, j) \in \mathcal{S}$. The entries of the additive noise $\mathbf{n}_{i, j} \in \mathbb{C}^{N_{r}}$ are independent and identically distributed (i.i.d) noise samples with zero mean and variance $\sigma^{2}$. The covariance of $\mathbf{y}_{i, j}$ is thus

$$
\mathbf{M}_{i, j}(\mathbf{V})=\sum_{(s, l) \in \mathcal{S}} \mathbf{H}_{s, i, j} \mathbf{V}_{s, l} \mathbf{V}_{s, l}^{H} \mathbf{H}_{s, i, j}^{H}+\sigma^{2} \mathbf{I}_{N_{r}} .
$$

Under the successive decoding and interference cancellation scheme described above, it follows that:

- UE $(i, j)$ can decode its own common message $\mathbf{s}_{i, j}^{c}$ with the achievable rate (expressed in nats/s/channel-use):

$$
r_{i, j}^{c}(\mathbf{V})=\ln \left|\mathbf{I}_{L}+\left(\mathbf{V}_{i, j}^{c}\right)^{H} \mathbf{H}_{i, i, j}^{H} \mathbf{M}_{i, j}^{c}(\mathbf{V})^{-1} \mathbf{H}_{i, i, j} \mathbf{V}_{i, j}^{c}\right|,
$$

where $\mathbf{M}_{i, j}^{c}(\mathbf{V}) \triangleq \mathbf{M}_{i, j}(\mathbf{V})-\mathbf{H}_{i, i, j} \mathbf{V}_{i, j}^{c}\left(\mathbf{V}_{i, j}^{c}\right)^{H} \mathbf{H}_{i, i, j}^{H}$. Accordingly, $\mathbf{V}_{i, j}^{c} \equiv \mathbf{0}, r_{i, j}^{c}(\mathbf{V}) \equiv 0$ and $\mathbf{M}_{i, j}^{c}(\mathbf{V}) \equiv \mathbf{M}_{i, j}(\mathbf{V})$ if $a(i, j)=\emptyset$.

- UE $(i, j)$ can decode the common message $\mathbf{s}_{\hat{i}, \hat{j}}^{c}$ from the interfering user $(\hat{i}, \hat{j})=a^{-1}(i, j)$ with the achievable rate:

$$
r_{i, j}^{a}(\mathbf{V})=\ln \left|\mathbf{I}_{L}+\left(\mathbf{V}_{\hat{i}, \hat{j}}^{c}\right)^{H} \mathbf{H}_{\hat{i}, i, j}^{H} \mathbf{M}_{i, j}^{a}(\mathbf{V})^{-1} \mathbf{H}_{\hat{i}, i, j} \mathbf{V}_{\hat{i}, \hat{j}}^{c}\right|,
$$

where $\mathbf{M}_{i, j}^{a}(\mathbf{V}) \triangleq \mathbf{M}_{i, j}^{c}(\mathbf{V})-\mathbf{H}_{\hat{i}, i, j} \mathbf{V}_{\hat{i}, \hat{j}}^{c}\left(\mathbf{V}_{\hat{i}, \hat{j}}^{c}\right)^{H} \mathbf{H}_{\hat{i}, i, j}^{H}$. Accordingly, $\mathbf{M}_{i, j}^{a}(\mathbf{V}) \equiv \mathbf{M}_{i, j}^{c}(\mathbf{V})$ if $a^{-1}(i, j)=\emptyset$.

- UE $(i, j)$ can decode its own private message $\mathbf{s}_{i, j}^{p}$ with the achievable rate:

$$
r_{i, j}^{p}(\mathbf{V})=\ln \left|\mathbf{I}_{L}+\left(\mathbf{V}_{i, j}^{p}\right)^{H} \mathbf{H}_{i, i, j}^{H} \mathbf{M}_{i, j}^{p}(\mathbf{V})^{-1} \mathbf{H}_{i, i, j} \mathbf{V}_{i, j}^{p}\right|,
$$

where $\mathbf{M}_{i, j}^{p}(\mathbf{V}) \triangleq \mathbf{M}_{i, j}^{a}(\mathbf{V})-\mathbf{H}_{i, i, j} \mathbf{V}_{i, j}^{p}\left(\mathbf{V}_{i, j}^{p}\right)^{H} \mathbf{H}_{i, i, j}^{H}$.

Similar to [7, (10)-(12)], the achievable rate region under the successive decoding is given by:

$$
\begin{aligned}
& \left\{\boldsymbol{v} \triangleq\left[v_{i, j}\right]_{(i, j) \in \mathcal{I} \times \mathcal{J}}=\left[v_{i, j}^{p}+v_{i, j}^{c}\right]_{(i, j) \in \mathcal{I} \times \mathcal{J}}:\right. \\
& \left.v_{i, j}^{p} \leq r_{i, j}^{p}(\mathbf{V}), v_{i, j}^{c} \leq r_{i, j}^{c}(\mathbf{V}), v_{i, j}^{c} \leq r_{a(i, j)}^{a}(\mathbf{V})\right\} .
\end{aligned}
$$

As $r_{a(i, j)}^{a}(\mathbf{V})$ is the achievable rate of decoding the common message $\mathbf{s}_{i, j}^{c}$ of UE $(i, j)$ by UE $(\tilde{i}, \tilde{j})=a(i, j)$, the constraint $v_{i, j}^{c} \leq r_{a(i, j)}^{a}(\mathbf{V})$ arises only when $a(i, j) \neq \emptyset$. Therefore, by defining the nonsmooth functions

$$
r_{i, j}(\mathbf{V})=r_{i, j}^{p}(\mathbf{V})+\min \left\{r_{i, j}^{c}(\mathbf{V}), r_{a(i, j)}^{a}(\mathbf{V})\right\},
$$

we formulate the problem of sum-rate maximization over the achievable rate region as:

$$
\begin{gathered}
\max _{\mathbf{V}} \mathcal{P}(\mathbf{V}) \triangleq \sum_{(i, j) \in \mathcal{S}} r_{i, j}(\mathbf{V}) \quad \text { s.t. } \\
\sum_{j \in \mathcal{J}}\left\langle\mathbf{V}_{i, j} \mathbf{V}_{i, j}^{H}\right\rangle \leq P_{i}^{\max }, \forall i \in \mathcal{I},
\end{gathered}
$$

where $P_{i}^{\max }$ is the maximum transmit power of BS $i$.

Problem (2) is a challenging nonconvex optimization problem because its objective function is nonconvex and even nonsmooth. It should be emphasized that the available sum-rate maximization solutions (see, e.g., [10]) are only suitable for the conventional coordinated precoding approach, which corresponds to $r_{i, j}(\mathbf{V}) \equiv r_{i, j}^{p}(\mathbf{V})$ and $\mathbf{V}=$ $\left[\mathbf{V}^{p}\right]_{(i, j) \in \mathcal{S}}$ (i.e., there is no split of UE's data). On the other hand, the covariance optimization approach in [8], [9] is applicable for the case of $L=N_{t}$ only, i.e., one independent 
data stream is sent per one transmit antenna. In this particular case, (2) can be equivalently transformed to the optimization of a d.c. function in the rank-free outer products $\mathbf{Q}_{i, j}^{x}=\mathbf{V}_{i, j}^{x}\left(\mathbf{V}_{i, j}^{x}\right)^{H} \geq \mathbf{0}, x \in\{p, c\}$. The computational complexity of each d.c. iteration in [8] is high, because it involves the maximization of a logarithmic-determinant function under semi-definite constraints-a difficult convex optimization problem with unknown polynomial computational complexity. Whenever $L<N_{t}$, such a variable change leads to the additional difficult rank constraints $\operatorname{rank}\left(\mathbf{Q}_{i, j}^{x}\right) \leq L$ for which there is no available solution method. Indeed, there is no effective d.c. representation of each rate function in (1) even for the simplest case of $L=1$.

In what follows, we will develop an efficient successive optimization algorithm of low computational complexity to solve problem (2). Our solution works for both cases of $L=N_{t}$ and $L<N_{t}$.

Remark. We adopt the pairing rule proposed in [7]. At the receiver of each UE, the optimal solution of (2) for $\mathbf{V}_{i, j}^{c} \equiv$ $\mathbf{0}$ is used to identify the interference power from the UEs of other cells. Then, each UE is paired with the UE from a different cell that introduces the strongest interference.

\section{Proposed Precoder Design}

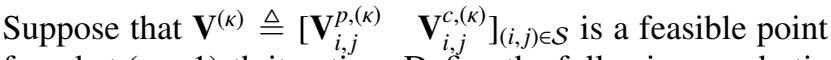
found at $(\kappa-1)$-th iteration. Define the following quadratic functions:

$$
\begin{gathered}
r_{i, j}^{c,(\kappa)}(\mathbf{V}) \triangleq r_{i, j}^{c}\left(\mathbf{V}^{(\kappa)}\right)+2 \mathfrak{R}\left\{\left\langle\mathcal{A}_{i, j}^{c,(\kappa)}, \mathbf{V}_{i, j}^{c}-\mathbf{V}_{i, j}^{c,(\kappa)}\right\rangle\right\} \\
-\left\langle\mathbf{M}_{i, j}^{c}\left(\mathbf{V}^{(\kappa)}\right)^{-1}-\mathbf{M}_{i, j}\left(\mathbf{V}^{(\kappa)}\right)^{-1},\right. \\
\left.\mathbf{M}_{i, j}(\mathbf{V})-\mathbf{M}_{i, j}\left(\mathbf{V}^{(\kappa)}\right)\right\rangle, \\
r_{i, j}^{a,(\kappa)}(\mathbf{V}) \triangleq r_{i, j}^{a}\left(\mathbf{V}^{(\kappa)}\right)+2 \mathfrak{R}\left\{\left\langle\mathcal{A}_{\hat{i}, \hat{j}}^{a,(\kappa)}, \mathbf{V}_{\hat{i}, \hat{j}}^{c}-\mathbf{V}_{\hat{i}, \hat{j}}^{c,(\kappa)}\right\rangle\right\} \\
-\left\langle\mathbf{M}_{i, j}^{a}\left(\mathbf{V}^{(\kappa)}\right)^{-1}-\mathbf{M}_{i, j}^{c}\left(\mathbf{V}^{(\kappa)}\right)^{-1},\right. \\
\left.\mathbf{M}_{i, j}^{c}(\mathbf{V})-\mathbf{M}_{i, j}^{c}\left(\mathbf{V}^{(\kappa)}\right)\right\rangle, \\
r_{i, j}^{p,(\kappa)}(\mathbf{V}) \triangleq r_{i, j}^{p}\left(\mathbf{V}^{(\kappa)}\right)+2 \mathfrak{R}\left\{\left\langle\mathcal{A}_{i, j}^{p,(\kappa)}, \mathbf{V}_{i, j}^{p}-\mathbf{V}_{i, j}^{p,(\kappa)}\right\rangle\right\} \\
-\left\langle\mathbf{M}_{i, j}^{p}\left(\mathbf{V}^{(\kappa)}\right)^{-1}-\mathbf{M}_{i, j}^{a}\left(\mathbf{V}^{(\kappa)}\right)^{-1},\right. \\
\left.\mathbf{M}_{i, j}^{a}(\mathbf{V})-\mathbf{M}_{i, j}^{a}\left(\mathbf{V}^{(\kappa)}\right)\right\rangle,
\end{gathered}
$$

where

$$
\begin{aligned}
& \mathcal{A}_{i, j}^{c,(\kappa)} \triangleq \mathbf{H}_{i, i, j}^{H} \mathbf{M}_{i, j}^{c}\left(\mathbf{V}^{(\kappa)}\right)^{-1} \mathbf{H}_{i, i, j} \mathbf{V}_{i, j}^{c,(\kappa)}, \\
& \mathcal{A}_{\hat{i}, \hat{j}}^{a,(\kappa)} \triangleq \mathbf{H}_{\hat{i}, i, j}^{H} \mathbf{M}_{i, j}^{a}\left(\mathbf{V}^{(\kappa)}\right)^{-1} \mathbf{H}_{\hat{i}, i, j} \mathbf{V}_{\hat{i}, \hat{j}}^{c,(\kappa)}, \\
& \mathcal{A}_{i, j}^{p,(\kappa)} \triangleq \mathbf{H}_{i, i, j}^{H} \mathbf{M}_{i, j}^{p}\left(\mathbf{V}^{(\kappa)}\right)^{-1} \mathbf{H}_{i, i, j} \mathbf{V}_{i, j}^{p,(\kappa)} .
\end{aligned}
$$

Note that all the above functions are concave in $\mathbf{V}$ because $\mathbf{M}_{i, j}^{c}\left(\mathbf{V}^{(\kappa)}\right)^{-1}-\mathbf{M}_{i, j}\left(\mathbf{V}^{(\kappa)}\right)^{-1} \geq \mathbf{0}, \mathbf{M}_{i, j}^{a}\left(\mathbf{V}^{(\kappa)}\right)^{-1}-\mathbf{M}_{i, j}^{c}\left(\mathbf{V}^{(\kappa)}\right)^{-1} \geq$ $\mathbf{0}, \mathbf{M}_{i, j}^{p}\left(\mathbf{V}^{(\kappa)}\right)^{-1}-\mathbf{M}_{i, j}^{a}\left(\mathbf{V}^{(\kappa)}\right)^{-1} \geq \mathbf{0}$.

The following result shows that the complicated function defined by (1) is lower bounded by a concave quadratic function.

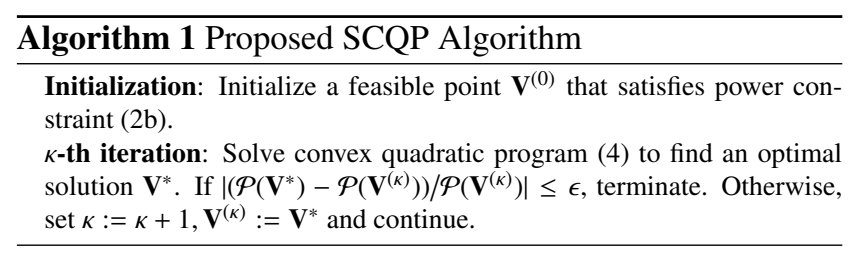

Theorem 1: For

$$
r_{i, j}^{(\kappa)}(\mathbf{V}) \triangleq r_{i, j}^{p,(\kappa)}(\mathbf{V})+\min \left\{r_{i, j}^{c,(\kappa)}(\mathbf{V}), r_{a(i, j)}^{a,(\kappa)}(\mathbf{V})\right\},
$$

it is true that

$$
r_{i, j}\left(\mathbf{V}^{(\kappa)}\right)=r_{i, j}^{(\kappa)}\left(\mathbf{V}^{(\kappa)}\right) \quad \text { and } \quad r_{i, j}(\mathbf{V}) \geq r_{i, j}^{(\kappa)}(\mathbf{V}), \forall \mathbf{V} .
$$

Proof. The proof is given in Appendix.

In Algorithm 1, we propose a successive convex quadratic programming (SCQP) algorithm to solve problem (2). Given a feasible point $\mathbf{V}^{(\kappa)}$, this algorithm iteratively generates a feasible point $\mathbf{V}^{(\kappa+1)}$ as the optimal solution to the following optimization problem at the $\kappa$-th iteration:

$$
\max _{\mathbf{V}} \mathcal{P}^{(\kappa)}(\mathbf{V}) \triangleq \sum_{(i, j) \in \mathcal{S}} r_{i, j}^{(\kappa)}(\mathbf{V}) \quad \text { s.t. } \quad(2 b) .
$$

Problem (4) is a convex quadratic with $m=N+2 K N$ quadratic constraints and $n=2 N K N_{t} L+K N$ real decision variables. The complexity for computing its optimal solution $\mathbf{V}^{(\kappa+1)}$ is thus $O\left(n^{2} m^{2.5}+m^{3.5}\right)$. Note that $\mathbf{V}^{(\kappa)}$ is also feasible to (4) with $\mathcal{P}\left(\mathbf{V}^{(\kappa)}\right)=\mathcal{P}^{(\kappa)}\left(\mathbf{V}^{(\kappa)}\right)$ by the equality in (3). It is then true that $\mathcal{P}^{(\kappa)}\left(\mathbf{V}^{(\kappa+1)}\right)>\mathcal{P}^{(\kappa)}\left(\mathbf{V}^{(\kappa)}\right)=\mathcal{P}\left(\mathbf{V}^{(\kappa)}\right)$ whenever $\mathbf{V}^{(\kappa+1)} \neq \mathbf{V}^{(\kappa)}$. Together with $\mathcal{P}\left(\mathbf{V}^{(\kappa+1)}\right) \geq \mathcal{P}^{(\kappa)}\left(\mathbf{V}^{(\kappa)}\right)$ according to the inequality in (3), we have that $\mathcal{P}\left(\mathbf{V}^{(\kappa+1)}\right)>\mathcal{P}\left(\mathbf{V}^{(\kappa)}\right)$, i.e., the optimal solution $\mathbf{V}^{(\kappa+1)}$ of the convex quadratic problem (4) is a better point of the nonconvex nonsmooth optimization problem (2) than $\mathbf{V}^{(\kappa)}$. Therefore, once initialized from an achievable sum-rate $\mathcal{P}\left(\mathbf{V}^{(0)}\right)$, the sequence $\left\{\mathcal{P}\left(\mathbf{V}^{(\kappa)}\right)\right\}$ obtained by solving (4) is guaranteed to improve at each iteration and it eventually converges to at least a local optimum of (2) [11].

\section{Numerical Results}

In our simulations, we assume $\mathbf{H}_{i, s, l} \triangleq \sqrt{\eta_{i, s, l}} \tilde{\mathbf{H}}_{i, s, l}$ where $\tilde{\mathbf{H}}_{i, s, l} \in \mathbb{C}^{N_{t} \times N_{r}}$ with $N_{t}=4$ and $N_{r}=2$ represents the normalized MIMO channel, the entries of which are independent and identically distributed complex Gaussian variables with zero-mean and unit variance. Following [4], [12] the direct channel powers $\eta_{i, i, l}$ are fixed, while the interfering channel powers $\eta_{i, j, \ell}(i \neq j)$ are varied to cover all the environment-dependent channel effects, including path loss and shadowing. The simulation scenarios thus vary from weak MIMO INs to mixed MIMO INs, for which the HanKobayashi strategy is advantageous. The notation ' $\mathrm{H}-\mathrm{K}$ ' refers to the Han-Kobayashi strategy whereas 'coordinated' refers to the conventional coordinated precoding approach which only involves private message precoding. It can be 


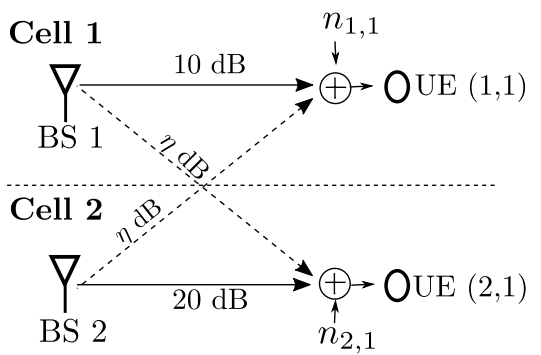

(a) $N=2, K=1$

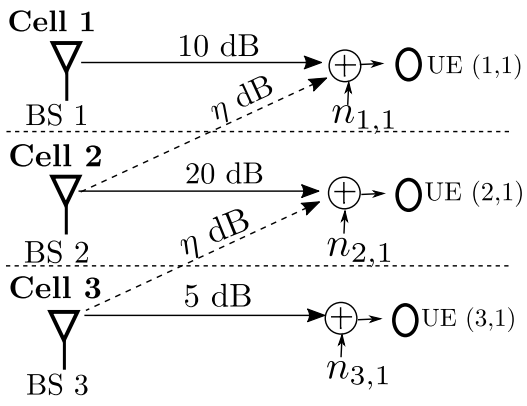

(b) $N=3, K=1$

Fig. 1 Network configurations used in the simulations.

seen from (2) that the IN sum rate monotonically increases in the number of involved data streams $L$. Since the covariance optimization approach in [8] is suitable for $L=N_{t}=4$, it is used for performance evaluation in this case. The comparison between the ' $\mathrm{H}-\mathrm{K}$ ' and 'coordinated' schemes is to show the capability of the H-K strategy in mitigating the intercell interferences. Each result in the Monte-Carlo simulation is obtained upon averaging over 100 random network realizations. We set the error tolerance as $\epsilon=10^{-4}$ and $\sigma^{2}=1, P_{i}^{\max }=10^{3}, \forall i$ in (2). We divide the achieved sumrate results by $\ln (2)$ to arrive at the unit of bps/channel-use for binary communications.

First, we consider the two-cell two-UE MIMO network depicted in Fig. 1(a). The values of direct channel gains $\eta_{1,1,1}$ and $\eta_{2,2,1}$ are indicated in the figure, while interference channel gains $\eta_{1,2,1}=\eta_{2,1,1}=\eta$ are varied from $-40 \mathrm{~dB}$ to $20 \mathrm{~dB}$. In this scenario, UEs do not experience any intracell interference and the only UE pairing possibilities are $a(1,1)=(2,1)$ and $a(2,1)=(1,1)$. Figure 2 also includes the curve of the theoretical lower bound and upper bound by solving the linear inequality [5, (52a)-(52i)] and [5, (11)(17)], respectively. While the performance of the H-K strategy with $L=4$ or $L=2$ is above the lower bound for the whole range of $\eta$ considered, the performance of the "coordinated' scheme is worse than the lower bound for $\eta \geq 10 \mathrm{~dB}$. More importantly, the H-K strategy in all considered cases of $L$ is able to achieve better sum rate performance even when the interference channel gain increases. As seen, the ' $\mathrm{H}-\mathrm{K}$ ' scheme offers a substantial performance gain over the 'coordinated' counterpart, especially for large interference channel gain $\eta$. In particular, an improvement of up to $30 \%$ is observed for $L=1$ and $\eta=20 \mathrm{~dB}$. In addition, the performance of the H-K strategy with $L=2$ and the proposed

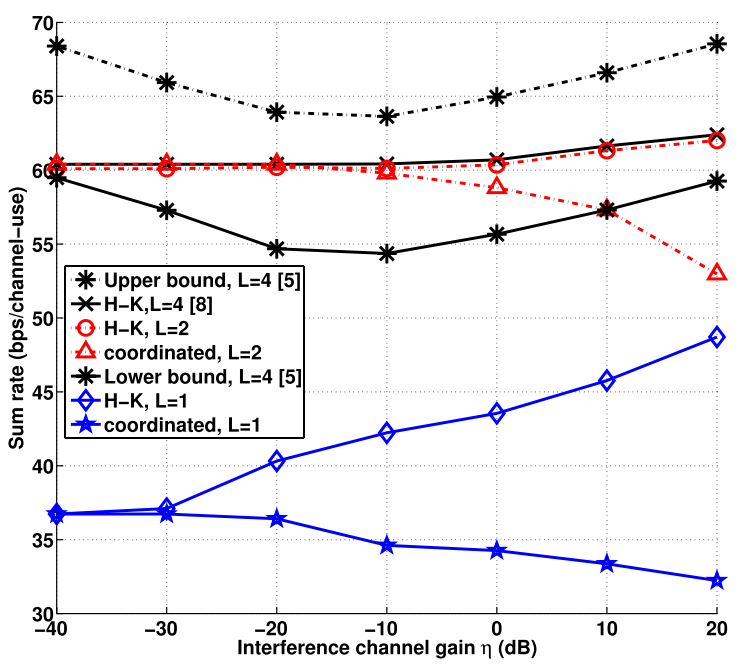

Fig. 2 Performance results for the network in Fig. 1(a).

Table 1 Average number of iterations by Algorithm 1 for the network in Fig. 1(a).

\begin{tabular}{|c|c|c|c|c|c|}
\cline { 3 - 6 } \multicolumn{2}{c|}{} & \multicolumn{4}{c|}{$\eta(\mathrm{dB})$} \\
\cline { 3 - 6 }$\epsilon=10^{-2}$ & -10 & 0 & 10 & 20 \\
\hline & Coordinated, $L=1$ & 5.6 & 3.1 & 2 & 2 \\
\cline { 2 - 6 } & $\mathrm{H}-\mathrm{K}, L=1$ & 5.6 & 3.2 & 2.2 & 2.3 \\
\cline { 2 - 6 } & Coordinated, $L=2$ & 5.7 & 3.2 & 2.1 & 2.6 \\
\cline { 2 - 6 }$\epsilon=10^{-3}$ & $\mathrm{H}-\mathrm{K}, L=2$ & 6.1 & 4.2 & 2.7 & 2.7 \\
\hline & Coordinated, $L=1$ & 5.5 & 2.5 & 2 & 2 \\
\cline { 2 - 6 } & $\mathrm{H}-\mathrm{K}, L=1$ & 15.2 & 20.7 & 15.6 & 23.6 \\
\cline { 2 - 6 } & Coordinated, $L=2$ & 23.88 & 15.5 & 10 & 7.1 \\
\cline { 2 - 6 }$\epsilon=10^{-4}$ & $\mathrm{H}-\mathrm{K}, L=2$ & 32.4 & 16.1 & 11.9 & 8.7 \\
\hline & Coordinated, $L=1$ & 8.1 & 6.7 & 8.8 & 15.5 \\
\cline { 2 - 6 } & $\mathrm{H}-\mathrm{K}, L=1$ & 28.1 & 35.2 & 35.2 & 41.3 \\
\cline { 2 - 6 } & Coordinated, $L=2$ & 43.8 & 31.8 & 34.1 & 27.1 \\
\cline { 2 - 6 } & $\mathrm{H}-\mathrm{K}, L=2$ & 41.5 & 55.3 & 34.6 & 38.1 \\
\hline
\end{tabular}

precoding matrices is very close to the performance of the scheme in [8] under the covariance matrix design and $L=4$. This means that our solution approaches the global optimum of problem (2).

Table 1 shows the average number of iterations required for Algorithm 1 to converge, which is similar to the convergence result in [8, Table IV] for the SDP-based covariance optimization algorithm. Since our proposed algorithm is an iterative procedure, the value of $\epsilon$ determines the number of iterations and the performance of the algorithm. In general, choosing which value of $\epsilon$ is governed the tradeoff between performance and complexity one wants to make. Our numerical results show that the performance of the proposed algorithm is indeed improved as $\epsilon$ decreases. For clarity of presentation, we only include the performance of the proposed algorithm with $\epsilon=10^{-4}$ and focus on performance comparison between the H-K strategy and the "coordinated' scheme. However, for all the values of $\epsilon$ considered, the H-K solutions outperform the solutions corresponding to the coordinated scheme.

Next, we consider the three-cell three-UE MIMO network depicted in Fig. 1(b). The values of direct channel gains are indicated in the figure. Following [8, Fig. 5(b)], 


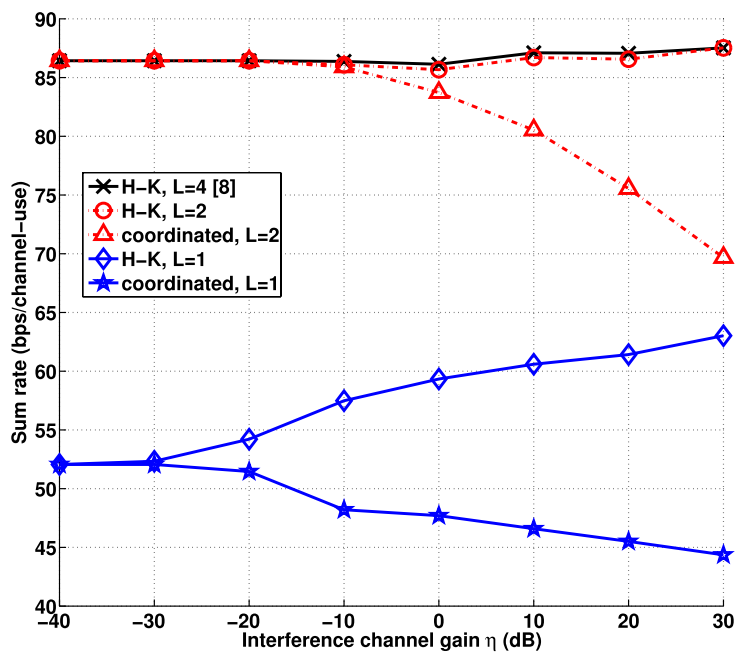

Fig. 3 Performance results for the network in Fig. 1(b).

we set $\eta_{1,2,1}=\eta_{2,3,1}=\eta_{3,1,1}=0$, while varying other interfering channel gains $\eta_{2,1,1}=\eta_{3,2,1}=\eta$ from $-40 \mathrm{~dB}$ to $30 \mathrm{~dB}$. In this case, the obvious choice for UE pairing is $a(1,1)=(2,1), a(2,1)=(3,1)$. Figure 3 demonstrates the $\mathrm{H}-\mathrm{K}$ strategy is again able to improve the sum-rate performance under stronger channel interferences for all cases of $L$. The performance gap between ' $\mathrm{H}-\mathrm{K}$ ' and 'coordinated' curves is widened especially in the high interference region of $\eta \geq 20 \mathrm{~dB}$.

It is worth noting that in both considered examples, the performances of ' $\mathrm{H}-\mathrm{K}$ ' scheme are not much distinguishable for $L=2$ and $L=4$, although the optimal covariance matrices $\mathbf{Q}_{i, j}^{x} \in \mathbb{C}^{4 \times 4}, x \in\{p, c\}$ are not necessarily of rank $N_{r}=2$ [8]. This result implies that using $L=2$ data streams gives a performance that is close to the best sum-rate performance. For $L=1$, an improvement is still observed in the region $\eta \in[-20,-10] \mathrm{dB}$, where the interference per antenna at each $\mathrm{UE}$ is at least $14.47 \mathrm{~dB}$ and $8.27 \mathrm{~dB}$ for the networks in Fig. 1(a) and Fig. 1(b), respectively. These levels of interference are well above the background noise power of $0 \mathrm{~dB}$. It is sufficient for the low interference regime condition to be satisfied only for the optimal input covariance matrices (those that maximize the achievable sum rate assuming Gaussian inputs and treating interference as noise) if they are full rank [13].

Remark 1: Since the focus of this work is to find the optimal precoding matrices under the $\mathrm{H}-\mathrm{K}$ strategy for a given user pairing choice, the considered simulation scenarios are limited to having one interferer. In the presence of multiple inter-cell interferers, the $\mathrm{H}-\mathrm{K}$ strategy only mitigates one paired source of inter-cell interference. The H-K strategy may not be better than the conventional scheme if there is no dominant interferer or if the pairing choices are not optimized. In general, the $\mathrm{H}-\mathrm{K}$ strategy is most efficient when a user has a dominant source of inter-cell interference. A similar result was obtained in [8] for the case of covariance matrices design.

\section{Conclusions}

This paper has addressed the problem of precoder design for both common and private messages in MU-MIMO multicell networks under the Han-Kobayashi strategy. Our aim is to find the optimal precoding matrices for network sumrate maximization. We have proposed a successive convex quadratic programming algorithm to solve the nonconvex optimization problem in the precoder matrices. Numerical results have confirmed the potential advantages of our proposed approach and also the ability of the Han-Kobayashi to mitigate the intercell interference, which leads to even better sum-rate despite an increase in channel interference.

\section{References}

[1] V. Annapureddy and V. Veeravalli, "Sum capacity of MIMO interference channels in the low interference regime," IEEE Trans. Inf. Theory, vol.57, no.5, pp.2565-2581, May 2011.

[2] X. Shang and H.V. Poor, "Noisy-interference sum-rate capacity for vector Gaussian interference channels," IEEE Trans. Inf. Theory, vol.59, no.1, pp.132-153, Jan. 2013.

[3] T.S. Han and K. Kobayashi, "A new achievable region for the interference channel," IEEE Trans. Inf. Theory., vol.33, no.1, pp.49-60, 1981.

[4] R. Etkin, D. Tse, and H. Wang, "Gaussian interference channel capacity to within one bit," IEEE Trans. Inf. Theory, vol.54, no.12, pp.5534-5562, Dec. 2008.

[5] S. Karmakar and M. Varanasi, "The capacity region of the MIMO interference channel and its reciprocity to within a constant gap," IEEE Trans. Inf. Theory, vol.59, no.8, pp.4781-4797, Aug. 2013.

[6] X. Shang, B. Chen, and M. Gans, "On the achievable sum rate for MIMO interference channels," vol.52, no.9, pp.4313-4320, Sept. 2006.

[7] H. Dahrouj and W. Yu, "Multicell interference mitigation with joint beamforming and common message decoding," IEEE Trans. Commun., vol.59, no.8, pp.2264-2273, Aug. 2011.

[8] E. Che, H.D. Tuan, H.H.M. Tam, and H. Nguyen, "Successive interference mitigation in multiuser mimo channels," IEEE Trans. Commun., vol.63, no.6, pp.2185-2199, June 2015.

[9] E. Che and H. Tuan, "Interference mitigation by jointly splitting rates and beamforming for multi-cell multi-user networks," Proc. 13th ISCIT, pp.41-45, Sept. 2013.

[10] S. Christensen, R. Agarwal, E. Carvalho, and J. Cioffi, "Weighted sum-rate maximization using weighted MMSE for MIMO-BC beamforming design," IEEE Trans. Wireless Commun., vol.7, no.12, pp.4792-4799, Dec. 2008.

[11] B.R. Marks and G.P. Wright, "A general inner approximation algorithm for nonconvex mathematical programms," Oper. Res., vol.26, no.4, pp.681-683, July 1978.

[12] R. Blum, "MIMO capacity with interference," IEEE J. Sel. Areas Commun., vol.21, no.5, pp.793-801, June 2003.

[13] V.S. Annapureddy and V.V. Veeravalli, "Sum capacity of mimo interference channels in the low interference regime," IEEE Trans. Inf. Theory, vol.57, no.5, pp.2565-2581, May 2011.

[14] U. Rashid, H.D. Tuan, H.H. Kha, and H.H. Nguyen, "Joint optimization of source precoding and relay beamforming in wireless MIMO relay networks," IEEE Trans. Commun., vol.62, no.2, pp.488-499, Feb. 2014.

[15] H. Tuy, Convex Analysis and Global Optimization, Kluwer Academic, 1998. 


\section{Appendix: Proof of Theorem 1}

Due to space limitation, we only provide an outline of this quite complicated proof. The equality in (3) is obvious because $r_{i, j}^{x}\left(\mathbf{V}^{(\kappa)}\right)=r_{i, j}^{x,(\kappa)}\left(\mathbf{V}^{(\kappa)}\right)$ for $x \in\{c, a, p\}$. To prove the inequality in (3), it suffices to show that

$$
r_{i, j}^{x}(\mathbf{V}) \geq r_{i, j}^{x,(\kappa)}(\mathbf{V}) \forall \mathbf{V}, x \in\{c, a, p\} .
$$

We will prove (A 1$)$ for $x=c$ only, as the proof for $x=a$ and $x=p$ is similar.

Define

$$
g\left(\mathbf{V}_{i, j}^{c}, \mathbf{M}\right) \triangleq \ln \left|\mathbf{I}_{L}-h\left(\mathbf{V}_{i, j}^{c}, \mathbf{M}\right)\right|
$$

and

$$
h\left(\mathbf{V}_{i, j}^{c}, \mathbf{M}\right) \triangleq\left(\mathbf{V}_{i, j}^{c}\right)^{H} \mathbf{H}_{i, i, j}^{H} \mathbf{M}^{-1} \mathbf{H}_{i, i, j} \mathbf{V}_{i, j}^{c}
$$

in the domain

$$
\mathcal{U} \triangleq\left\{\left(\mathbf{V}_{i, j}^{c}, \mathbf{M}\right): \mathbf{M}>\mathbf{H}_{i, i, j} \mathbf{V}_{i, j}^{c}\left(\mathbf{V}_{i, j}^{c}\right)^{H} \mathbf{H}_{i, i, j}^{H}\right\} .
$$

By [14, Appendix C], $h(.$, .) is convex-valued in $\mathcal{U}$, i.e.,

$$
\begin{aligned}
& \alpha h\left(\mathbf{V}_{i, j}^{c}, \mathbf{M}\right)+\beta h\left(\mathbf{V}_{i, j}^{c,(\kappa)}, \mathbf{M}^{(\kappa)}\right) \quad \geq \\
& h\left(\alpha\left(\mathbf{V}_{i, j}^{c}, \mathbf{M}\right)+\beta\left(\mathbf{V}_{i, j}^{c,(\kappa)}, \mathbf{M}^{(\kappa)}\right)\right),
\end{aligned}
$$

for all $\alpha \geq 0, \beta \geq 0, \alpha+\beta=1$. It follows that

$$
\begin{array}{ll}
g\left(\alpha\left(\mathbf{V}_{i, j}^{c}, \mathbf{M}\right)+\beta\left(\mathbf{V}_{i, j}^{c,(\kappa)}, \mathbf{M}^{(\kappa)}\right)\right) & = \\
\ln \left|\mathbf{I}_{N_{r}}-h\left(\alpha\left(\mathbf{V}_{i, j}^{c}, \mathbf{M}\right)+\beta\left(\mathbf{V}_{i, j}^{c,(\kappa)}, \mathbf{M}^{(\kappa)}\right)\right)\right| & \geq \\
\ln \left|\mathbf{I}_{N_{r}}-\left(\alpha h\left(\mathbf{V}_{i, j}^{c}, \mathbf{M}\right)+\beta h\left(\mathbf{V}_{i, j}^{c,(k)}, \mathbf{M}^{(\kappa)}\right)\right)\right| & \geq \\
\alpha \ln \left|\mathbf{I}_{N_{r}}-h\left(\mathbf{V}_{i, j}^{c}, \mathbf{M}\right)\right|+\beta \ln \left|\mathbf{I}_{N_{r}}-h\left(\mathbf{V}_{i, j}^{c,(\kappa)}, \mathbf{M}^{(\kappa)}\right)\right| & = \\
\alpha g\left(\mathbf{V}_{i, j}^{c}, \mathbf{M}\right)+\beta g\left(\mathbf{V}_{i, j}^{c,(\kappa)}, \mathbf{M}^{(\kappa)}\right), &
\end{array}
$$

i.e. $g(.$, .) is concave in $\mathcal{U}$.

Based on the fact that the first order approximation of a concave function at a given point is its global upper bound [15], we have

$$
g\left(\mathbf{V}_{i, j}^{c}, \mathbf{M}\right) \leq g^{(\kappa)}\left(\mathbf{V}_{i, j}^{c}, \mathbf{M}\right)
$$

where using a standard differential calculus of log-det functions yields

$$
\begin{aligned}
& g^{(\kappa)}\left(\mathbf{V}_{i, j}^{c}, \mathbf{M}\right) \\
& g\left(\mathbf{V}_{i, j}^{c,(\kappa)}, \mathbf{M}^{(\kappa)}\right) \\
& +\left\langle\nabla g\left(\mathbf{V}_{i, j}^{c,(\kappa)}, \mathbf{M}^{(\kappa)}\right),\left(\mathbf{V}_{i, j}^{c}, \mathbf{M}\right)-\left(\mathbf{V}_{i, j}^{c,(\kappa)}, \mathbf{M}^{(\kappa)}\right)\right\rangle= \\
& g\left(\mathbf{V}_{i, j}^{c,(\kappa)}, \mathbf{M}^{(\kappa)}\right) \\
& -2 \Re\left\{\left\langle\mathbf{H}_{i, i, j}^{H}\left(\mathbf{M}^{(\kappa)}\right)^{-1} \mathbf{V}_{i, j}^{c,(\kappa)} \mathbf{H}_{i, i, j}, \mathbf{V}_{i, j}-\mathbf{V}_{i, j}^{c,(\kappa)}\right\rangle\right\} \\
& +\left\langle\left(\mathbf{M}^{(\kappa)}-\mathbf{H}_{i, i, j} \mathbf{V}_{i, j}^{c,(\kappa)}\left(\mathbf{V}_{i, j}^{c,(\kappa)}\right)^{H} \mathbf{H}_{i, i, j}^{H}\right)^{-1}\right. \\
& \left.-\left(\mathbf{M}^{(\kappa)}\right)^{-1}, \mathbf{M}-\mathbf{M}^{(\kappa)}\right\rangle,
\end{aligned}
$$

Then (A. 1) for $x=c$ follows by substituting $\mathbf{M}=\mathbf{M}_{i, j}(\mathbf{V})$ and $\mathbf{M}^{(\kappa)}=\mathbf{M}_{i, j}\left(\mathbf{V}^{(\kappa)}\right)$ in (A.2) and verifying that

$$
r_{i, j}^{c}(\mathbf{V})=-g\left(\mathbf{V}_{i, j}^{c}, \mathbf{M}_{i, j}(\mathbf{V})\right)
$$

and

$$
r_{i, j}^{c,(\kappa)}(\mathbf{V})=-g^{(\kappa)}\left(\mathbf{V}_{i, j}^{c}, \mathbf{M}_{i, j}(\mathbf{V})\right) .
$$

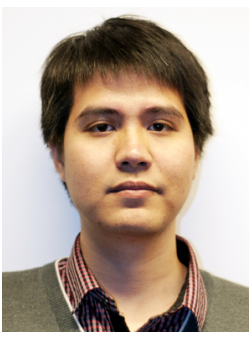

Ho Huu Minh Tam was born in Ho Chi Minh City, Vietnam. He received the B.S. degree in electrical engineering and telecommunications from the Ho Chi Minh City University of Technology, in 2012. He is currently pursuing the Ph.D. degree with the University of Technology Sydney, Australia, under the supervision of Prof. Hoang Duong Tuan. His research interest is in optimization techniques in signal processing for wireless communications.

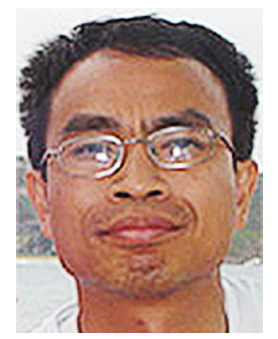

Hoang Duong Tuan received the Diploma (Hons.) and Ph.D. degrees in applied mathematics from Odessa State University, Ukraine, in 1987 and 1991, respectively. He spent nine academic years in Japan as an Assistant Professor with the Department of Electronic-Mechanical Engineering, Nagoya University, from 1994 to 1999, and then as an Associate Professor with the Department of Electrical and Computer Engineering, Toyota Technological Institute, Nagoya, from 1999 to 2003 . He was a Professor with the School of Electrical Engineering and Telecommunications, University of New South Wales, from 2003 to 2011 . He is currently a Professor with the Centre for Health Technologies, University of Technology Sydney. He has been involved in research with the areas of optimization, control, signal processing, wireless communication, and biomedical engineering for more than 20 years.

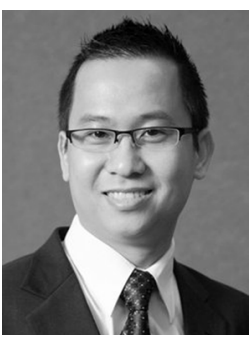

Duy Trong Ngo received the B.Eng. (with First-class Honours and the University Medal) degree in telecommunication engineering from The University of New South Wales, Australia, in 2007, the M.Sc. degree in electrical engineering (communication) from the University of $\mathrm{Al}-$ berta, Canada, in 2009, and the Ph.D. degree in electrical engineering from McGill University, Canada, in 2013. He has been a Lecturer with the School of Electrical Engineering and Computer Science, The University of Newcastle, Australia, since 2013. His current research interest is in the design and optimization of $5 \mathrm{G}$ wireless communications systems. 


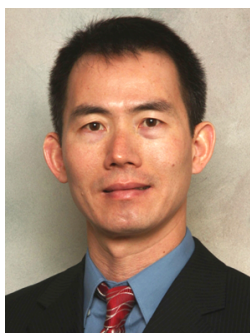

Ha Hoang Nguyen received the B.Eng. degree from the Hanoi University of Technology (HUT), Hanoi, Vietnam, in 1995, the M.Eng. degree from the Asian Institute of Technology (AIT), Bangkok, Thailand, in 1997, and the $\mathrm{Ph} . \mathrm{D}$. degree from the University of Manitoba, Winnipeg, MB, Canada, in 2001, all in electrical engineering. He joined the Department of Electrical and Computer Engineering, University of Saskatchewan, Saskatoon, SK, Canada, in 2001, and became a full Professor in 2007. He holds adjunct appointments at the Department of Electrical and Computer Engineering, University of Manitoba, Winnipeg, MB, Canada, and was a Senior Visiting Fellow in the School of Electrical Engineering and Telecommunications, University of New South Wales, Sydney, Australia. His research interests fall into broad areas of Communication Theory, Wireless Communications, and Statistical Signal Processing. Dr. Nguyen was an Associate Editor for the IEEE Transactions on Wireless Communications during 2007-2011. He currently serves as an Associate Editor for the IEEE Transactions on Vehicular Technology and the IEEE Wireless Communications Letters. He is a coauthor, with Ed Shwedyk, of the textbook "A First Course in Digital Communications" (published by Cambridge University Press). Dr. Nguyen is a Fellow of the Engineering Institute of Canada (EIC) and a Registered Member of the Association of Professional Engineers and Geoscientists of Saskatchewan (APEGS). 